\title{
Assessment Of Stress On Serum Estradiol Levels In Female Subordinate Naked Mole Rats Following Isolation From Natal Colony
}

AMOS ONYANSI MAKORI ( $\sim$ msmakori@gmail.com )

kisii university
A. W. NYONGESA
UNIVERSITY OF NAIROBI
H. ODONGO
university of nairobi

\section{R. J. MASAI}
Kisii University

\section{Research note}

Keywords: Isolation, Subordinate naked mole rat, Reproductive Success, Stress, Estradiol

Posted Date: September 24th, 2019

DOI: https://doi.org/10.21203/rs.2.14841/v1

License: (a) (i) This work is licensed under a Creative Commons Attribution 4.0 International License. Read Full License

Version of Record: A version of this preprint was published at Journal of Biosciences and Medicines on January 1st, 2020. See the published version at https://doi.org/10.4236/jbm.2020.83002. 


\section{Abstract}

Objective: This study investigated the effects of isolation of subordinate naked mole rats from natal colonies on their reproductive success. The study aimed at establishing whether the reproductive suppression in subordinate naked mole rats is the outcome of social stress exerted by breeding female in the colony or other unknown environmental factors within the colony set-up. Blood samples from experimental and control groups were collected on 2nd, 4th, 6th , 8th, 12th and 20th weeks of experimental period for hormonal analysis using ELISA technique. The Statistical analysis done using student t-test at $5 \%$ significance level. Results: Hormone analysis showed significant change in both cortisol $(t=8.74, P=0.01)$ and estradiol $(t=7.15, P=0.02)$ of subordinate isolated females. Results showed no correlation between stress and cyclicity. Conclusively, the observed reproductive suppression among subordinate naked mole rats in natal colonies is probably due to presence of queen or other reproductive aspects that may not be directly related to stress.

\section{Background}

Naked mole rats (Heterocephalus glaber) are found in many parts of Makueni and Kitui Counties, in Kenya. Their reproduction is restricted to one queen and less than three males; the rest of the animals within the colony remain sterile. The proximate mechanisms of reproductive skew observed among the cooperative breeders have been studied. For instance, in Marmoset monkeys, a combination of behavioural and pheromonal signals from the dominant female bring about insufficient secretions of gonadotrophic hormones in other females [1]. Similar studies on Meerkats showed that physical aggression by dominant breeder towards the subordinate females was responsible for reproductive suppression[2]. The factors underlying reproductive inhibition among subordinate female naked mole rats in a natal colony remain obscure. Whether or not this inhibition results from social stress exerted by the breeding female, preference by breeding males to the queen over subordinate females or due to other ecological factors remain to be elucidated. The present study, therefore, interfered with the natal colony set up in order to explore the possibility that reproductive suppression on the female subordinate naked mole rats could be a form of stressor that impairs cyclicity, reproductive hormone synthesis and secretion and, therefore, reproductive success. Since the study considered isolation to be a form of stress, eusociality of the naked mole rats made them ideal animal model for the present study. The findings of this study would provide the basis for studying variations in reproductive hormones which could be applicable to human beings when exposed to stress and how these contribute to trade-offs with reproduction.

\section{Methods}

\subsection{Animal capture, sexing and Housing}

Forty naked mole rats were captured by simple random sampling technique from different natal colonies through live traps method. Sexing was done before introduction of the animals into the artificial colony. 
Each animal was examined around the genital area, between the two orifices, for the presence of a horizontal red line, a characteristic anatomical feature for females. The animals were marked with a tattoo ink for easy identification since they are homomorphic in appearance. Captive colony was made in artificial burrow system consisting of small cubical Plexiglas chambers $(20 \mathrm{~cm} \times 20 \mathrm{~cm} \times 20 \mathrm{~cm})$ linked by transparent glass tubing of $3 \mathrm{~m}$ tunnel system in a dimly lit room maintained at approximately $26^{\circ} \mathrm{C}$ room temperature with humidity of approximately $60 \%$. The animal house was cleaned daily and food supplied at the opening of the cubical boxes daily. Beddings for the animals were dry treated sawdust.

\subsection{Experimental design}

This comprised of three groups: two for experimental groups (singly isolated females (SIF) and female paired with males (FIP)) and non-isolated females (NIF) as controls. The experiment was carried out in two phases: acclimatization and isolation

\subsubsection{Acclimatization}

Before acclimatization, 2 female subordinate naked mole rats were sacrificed. Blood samples were collected and appropriately preserved for later analysis. On the $2^{\text {nd }}, 4^{\text {th }}$ and $6^{\text {th }}$ weeks of acclimatization, 2 female mole rats were sacrificed at each stage and blood samples, were harvested for analysis. Acclimatization covered the period between day 1 and week 6 of study while all animals were still in parent colony.

\subsubsection{Isolation from parent colony}

Isolation was done on week 7 of experimental period. Eighteen subordinate females were isolated and randomly assigned into two groups of 9 animals each in two separate cages for 14 weeks. One week 8, 2 subordinate females from each group were randomly selected, sacrificed and blood samples collected for analysis. The same procedure was repeated on week 12 and 20 of study.

\subsection{Animal euthanasia}

To ensure humane death, isoflurane was administered through inhalation using soaked cotton wool for $30 \mathrm{~min}$ in fuming chamber followed by decapitation.

\subsection{Blood Sampling}

Blood sample was collected using a 23-G needle directly from the apex of left ventricle after thoracotomy following anaesthesia with isoflurane (Sundent Pharmaceutical Co., Ltd, Shanghai, China) in a sealed chamber. About $500 \mu \mathrm{l}$ of blood sample was collected into red-topped vacuitainers followed by 
centrifugation at $1000 \mathrm{rpm}$ and serum decanted into clearly labelled ependorff tubes (by animal identity and date of collection) and subsequently frozen at $-20^{\circ} \mathrm{C}$ until hormone analysis.

\subsection{Hormone Analysis}

\subsubsection{Cortisol assay}

Serum cortisol was assayed using ELISA technique according to the manufacturer's protocol.The technique applies the principle of competition for limited binding sites on the specific antibody by both the hormone in sample and enzyme-conjugated hormone in the coated plates. First, the serum samples were thawed and homogenized by centrifugation. Twenty five microliters of the standards, sample and quality controls were first pippeted into the microtitre plate wells. Then $200 \mu \mathrm{l}$ of Enzyme Antigen Conjugate was added and mixture incubated for $60 \mathrm{~min}$ at $25^{\circ} \mathrm{C}$ followed by aspiration of contents and addition of $400 \mu \mathrm{l}$ of Wash solution. The washing procedure was repeated twice with a soak time of 30 sec before aspiration of the wash solution. Then $100 \mu \mathrm{l}$ of the substrate solution was added and the mixture incubated for $15 \mathrm{~min}$ at $25^{\circ} \mathrm{C}$. Then $100 \mu$ of the Stop solution was added followed by determination of absorbance at wavelength of $450 \mathrm{~nm}$ within $30 \mathrm{~min}$. The assay reagents (quality controls, standards, blanks and samples) were done in duplicate. The optical density of specimen was measured using HumaReader HS equipped with 450nm filters (Gessellschaft für Biochem und Diagnostica, $\mathrm{mbH}$, Germany).The assay sensitivity for cortisol was $1.1-1.5 \mathrm{ng} / \mathrm{ml}$. The intra -assay coefficient of variation was less than $15 \%$ while the inter assay coefficient of variation was less than $10 \%$.

\subsubsection{Estradiol assay}

Assay was also done by ELISA as per the protocol. After thawing reagents and specimen, twenty five microliters of the calibrators, specimen and controls were first pipetted into the micro plate wells. Then $100 \mu \mathrm{l}$ of the Enzyme Antigen Conjugate was added and vortex mixed. The mixture was incubated for 60 min at $25^{\circ} \mathrm{C}$. Then all the contents were aspirated followed by addition of $400 \mu$ of Wash solution. Washing procedure was repeated twice with a soak time of $30 \mathrm{sec}$ before aspiration of the wash solution. Then $100 \mu \mathrm{l}$ of the substrate solution was added and the mixture incubated for $15 \mathrm{~min}$ at $25^{\circ} \mathrm{C}$. Then $50 \mu \mathrm{l}$ of Stop solution was added and mixed carefully followed by measurement of absorbance at $450 \mathrm{~nm}$ within $10 \mathrm{~min}$. The assay procedure was also done in duplicate. The optical density of specimen was measured using HumaReader HS equipped with $450 \mathrm{~nm}$ filters. The assay sensitivity for estradiol was $13 \mathrm{pg} / \mathrm{ml}$. The intra -assay coefficient of variation was less than $15 \%$ while the inter assay coefficient of variation was less than $10 \%$.

\subsection{Statistical analysis}

The quantitative data on hormonal levels were analysed with IBM SPSS statistics version 20 yielding both descriptive and inferential statistical data. Data on hormonal levels are presented as mean \pm S.E.M. 
For parametric data the statistical differences between groups were determined by paired t-test. All the tests were two-tailed t-tests and the significance level was set at $P<0.05$.

\subsection{Quality Control}

To ensure validity on the data for hormone assay, the controls were assayed with a calibration curve and the quality control samples were run in triplicate alongside samples and standards and the mean values obtained were within the acceptable ranges. The pipetting was done quickly and with precision of the aliquots to avoid assay drift that could influence reliability of the results. The intra assay coefficients of variation were $3.10 \%$ for cortisol and $11.89 \%$ for estradiol.

\section{Results}

\subsection{Effects of colony re-organization on serum cortisol}

The mean serum cortisol levels during acclimation decreased significantly from 117.7 to $35.5 \mathrm{ng} / \mathrm{ml}$ from day 1 to week $6(t=3.49, P=0.04)$. After acclimation, there was $5.5 \%$ increase in the serum cortisol between week 8 and 1 week 12 in SIF. The increase in mean cortisol levels in SIF was greatest at the time of isolation from $35.5 \mathrm{ng} / \mathrm{ml}$ to $123.4 \mathrm{ng} / \mathrm{ml}$ compared to levels between week 8 and week 12 , whereby the hormone increased from 123.4 to $130.2 \mathrm{ng} / \mathrm{ml}$. However, there was $7.1 \%$ percentage decrease in serum cortisol in NIF. Similarly, in FIP, there was 9.9\% decrease in serum cortisol levels between week 8 and 12 . By week 20 mean serum cortisol levels in SIF had decreased significantly from 130.2 to $88.2 \mathrm{ng} / \mathrm{ml}(\mathrm{t}=$ 8.74, $\mathrm{P}=0.01)$, NIF from 108.1 to $90.4 \mathrm{ng} / \mathrm{ml}(\mathrm{t}=19.48, \mathrm{P}=0.01)$ and FIP from 100.2 to $87.4 \mathrm{ng} / \mathrm{ml}(\mathrm{t}=23.92$, $P=0.002)$. However, there was no significant difference in cortisol levels between the SIF and NIF ( $t=1.54$, $\mathrm{P}=0.263$ ) as well as FIP and NIF during this period. (Fig 1).

\subsection{Effects of colony re-organization on serum estradiol in females}

The serum estradiol levels increased significantly during acclimation with a mean of $14.5 \pm 0.96 \mathrm{pg} / \mathrm{ml}$ $(t=15.15, P=0.001)$. The SIF had $6.3 \%$ decrease in serum estradiol level between week 8 and 12 compared to NIF with $14.4 \%$ increase while FIP had $1.9 \%$ increase in serum estradiol levels. By week 20 , the SIF had a significant increase in Estradiol levels with mean values of $18 \pm 2.52 \mathrm{pg} / \mathrm{ml}(\mathrm{t}=7.15, \mathrm{P}=0.02)$ compared to NIF with mean of $15 \pm 0.58 \mathrm{pg} / \mathrm{ml}$. The mean serum estradiol levels in FIP also increased significantly from 16.1 to $25.0 \mathrm{pg} / \mathrm{ml}$ ( $\mathrm{t}=6.33, \mathrm{P}=0.02)$. There was no significant difference in mean estradiol levels between the SIF and FIP (paired $t=-1.73, P=0.23$ ). (Fig 2). 
Results showed no significant correlation between cortisol and estradiol levels during acclimation $(r=-0.755, P=0.245)$. After isolation, there was significant negative correlation in isolated females $(r=-0.999, P=0.023)$ but insignificant correlation in females paired with males $(r=-0.677, P=0.526)$ and the non-isolated females.

\section{Discussion}

Following isolation, the plasma cortisol levels in all the groups were generally higher compared to levels during acclimatization period implying that interference with social organisation by capture and isolation induced stress in the naked mole rats. Similar studies showed that repeated acute stress caused an increase in cortisol, as well as reproductive hormones in Wistar rats [3]. The lower mean serum estradiol levels in female subordinate naked mole rats between day 1 to week 6 compared to the levels measured during isolation period agrees with the previous studies [4] where female naked mole rats exhibited increased estradiol and progesterone levels immediately after one month of removal from their colony. There was accompanying increased steroid hormone receptors in the female breeders compared to nonbreeding female subordinate naked mole rats. This was indicative of possible reproductive suppression that led to delayed puberty in female subordinate naked mole rats. In another similar study[5], low urinary oestrogen in the non-breeding females was reported, confirming lack of ovarian cyclicity and ovulation. The lack of ovulation in non-breeding females was ascribed to inadequate LH concentration reported earlier[6]. Results of the present study showed more significant changes in estradiol levels in both ISF and IPF by week 20, indicating a possible transformation in reproductive status of the female subordinate females. In the present study there was no established relationship between the cortisol and the Estradiol levels. This finding is at variance with a closely related research finding done on the Sprague dowry rats [7] where it was reported that increased serum cortisol levels during exercise led to a decrease in serum progesterone which indicated possible suppression of reproduction by increased cortisol levels- a clear indicator of physiological stress. This finding is also similar to that reported in another study where levels of oestrogen and progesterone were shown to decrease as the responsiveness to stress increased[8]. In a previous study (Karsch et al., 2002), it was reported that interference of pulsatile release of LH is mediated by pathways that include the synthesis of prostaglandins and cortisol. The cortisol-mediated pathway reinforced the disruption, involved mostly in suppression of cyclicity during stressful events that cause activation of the HPA axis.

In conclusion, reproductive suppression of subordinate female can be ascribed to the presence of the queen or other reproductive aspects that may not be directly related to stress.

\section{Limitations}


- Other reproductive hormones (progesterone and luteinizing hormones) that could possibly vary as a result of isolation were not considered.

- The present study did not consider vaginal smear instead it only focussed on oestrogen levels as a marker of cyclicity.

\section{Declarations}

\section{Ethical Approval}

The research was approved by Biosafety, Animal use and Ethics committee, (REF: FAV BAUEC/2017/128) University of Nairobi and the animals were handled as directed by the committee using appropriate personal protective equipment (PPE). (Additional file 1)

\section{Availability of data and materials}

All data generated or analysed during this study are included in this published article and its supplementary information files. (Additional file 3 and 4 ).

\section{Funding}

The research work was funded by a research grant from National Research Fund (NRF) that made it possible to acquire kits for hormonal analysis and other laboratory reagents and equipment.

\section{Acknowledgements}

We acknowledge the Kenya wildlife services for granting us permit to capture naked mole rats for research (Additional file 2). We also acknowledge the Government of Kenya for funding our research through National Research Fund.

\section{Consent for publication}

Not applicable

\section{Competing interests}


The authors declare that they have no competing interests.

\section{Author's contributions}

A.O.M, A.W.N and R.J.M were the major contributors in writing the manuscript. H.O and A.O.M did hormonal analysis. All authors read and approved the final manuscript.

\section{List of additional files}

1. Ethical Approval Letter.

2. Animal Capture Permit.

3. Cortisol data

4. Estradiol data

\section{References}

[1] D. H. Abbott, W. Saltzman, and N. J. Schultz-darken, "Adaptations to subordinate status in female marmoset monkeys," Comp. Biochem. Physiol., vol. 119, pp. 261-274, 1998.

[2] T. H. Clutton-brock, S. J. Hodge, T. P. Flower, G. F. Spong, and A. J. Young, "Adaptive Suppression of Subordinate Reproduction in Cooperative Mammals," Am. Nat., vol. 176, no. 5, 2010.

[3] L. A. Olayaki and S. O. Suleiman, "Repeated acute restraint-induced stress:effects on body weight cortisol and reproductive hormones in female Wister rats," FASEB J., vol. 27, no. 1, p. 90, 2013.

[4] A. Swift-gallant, K. Mo, D. E. Peragine, D. A. Monks, and M. M. Holmes, "Removal of reproductive suppression reveals latent sex differences in brain steroid hormone receptors in naked mole-rats, Heterocephalus glaber," Biol. Sex Differ., pp. 1-9, 2015.

[5] P. Hagemeyer, S. Lange, M. Broecker-preuss, and H. Burda, "The influence of olfactory stimulus and sexual activity on gonadal steroids in eusocial mole-rats," Folia Zool., vol. 58, no. 1, pp. 65-74, 2009.

[6] C. . Faulkes, D. . . Abbott, and J. . . . Jarvis, "Social suppression of ovarian cyclicity in captive and wild colonies of naked mole-rats, Heterocephalus glaber," J. Reprod. Fertil., vol. 88, pp. 559-568, 1990.

[7] M. Mosavat, F. K. Ooi, and M. Mohamed, "Stress Hormone and Reproductive System in Response to Honey Supplementation Combined with Different Jumping Exercise Intensities in Female Rats," vol. 2014, 2014.

[8] T. A. Lovick, "Estrous cycle and stress: influence of progesterone on the female brain Estrous cycle and stress: influence of progesterone on the female brain," J. Med. Biol. Res., vol. 45, no. April, 2012. 


\section{Figures}

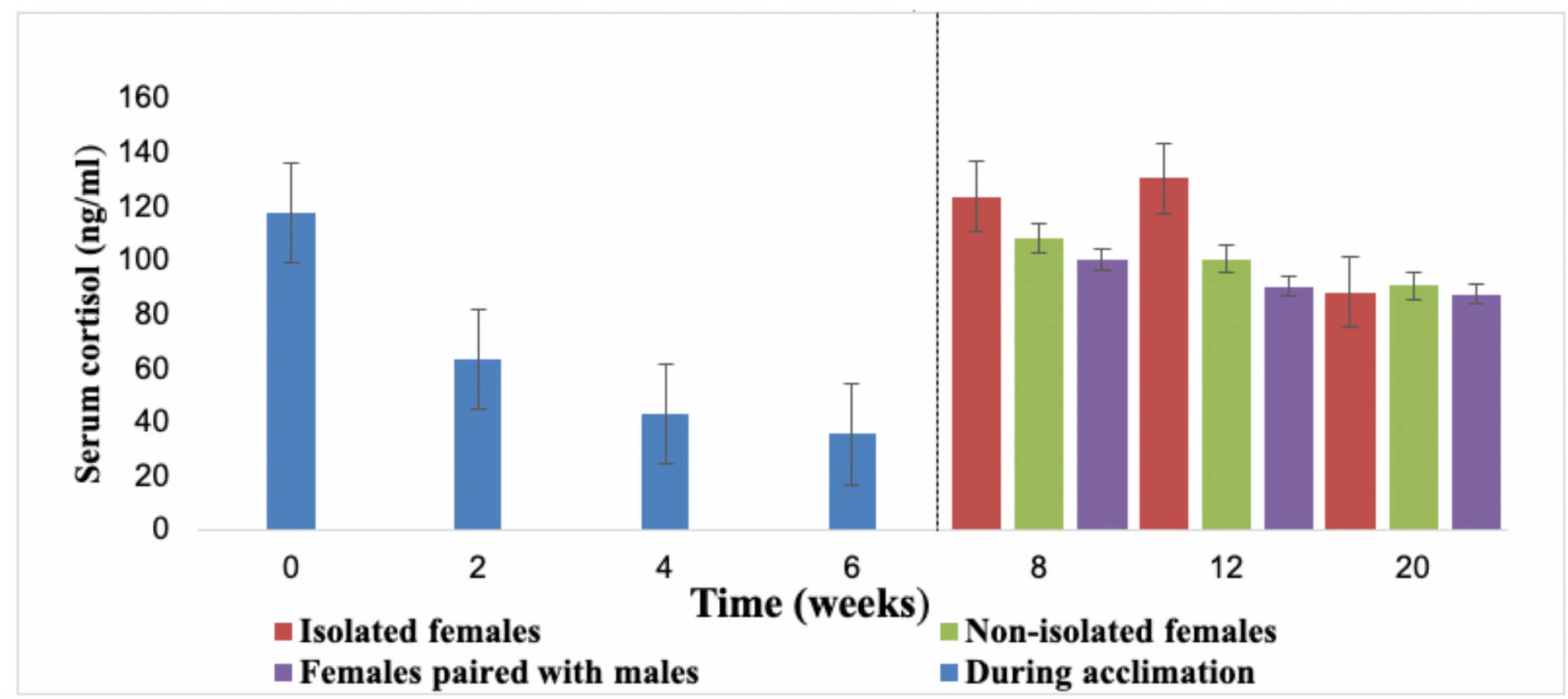

Figure 1

Pattern of mean serum cortisol measured over 20 weeks with the first 6 weeks showing hormone values during acclimation period while from week 8 to week 20 denotes period of re-grouping to isolated, nonisolated and pairing as shown in the key above. The dotted line denotes the time when isolation was done. $(n=26)$. 


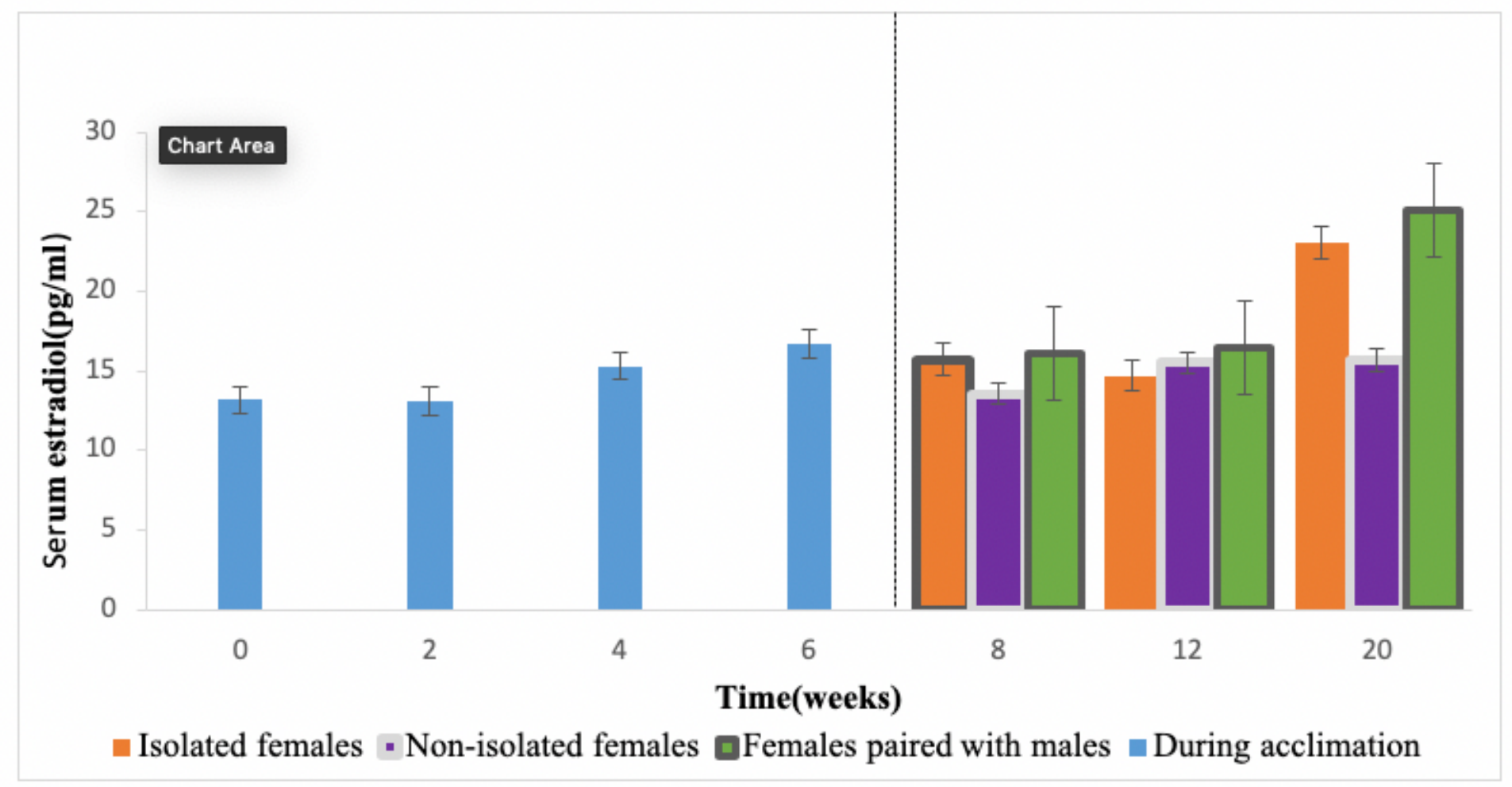

Figure 2

Mean serum estradiol levels of naked mole rats measured over 20 week experimental period. The vertical dotted line separates acclimation phase from isolation phase of study. Note the significant increase in serum estradiol among paired groups and isolated females by 20th week compared to non-isolated control.

\section{Supplementary Files}

This is a list of supplementary files associated with this preprint. Click to download.

- NC3RsARRIVEGuidelinesChecklistfillable.pdf

- capturepermit.jpeg

- ESTRADIOLDATA.xIsx 